\title{
E-motivate: Development of an App To Improve African Americans' Screening Colonoscopy Rates
}

Sarah Miller; Ashish Atreja; Farah Fasihuddin; Karthik Ramireddy; Rivelle Zlatopolsky; Allyssa Deorocki; Neda Hassanzadeh; Ariana Farahani; Sameed Lodhi; Emamuzo Otobo; Jason Rogers; Lina Jandorf

Icahn School of Medicine at Mount Sinai, New York, NY, United States

Corresponding Author:

Sarah Miller

Icahn School of Medicine at Mount Sinai

1468 Madison Avenue

New York, NY,

United States

Phone: 12122415090

Email: sarah.miller@mssm.edu

\section{Abstract}

Background: Colorectal cancer (CRC) is the third leading cause of death due to cancer in the United States. Compared to other racial/ethnic groups, African Americans have the highest CRC morbidity and mortality rates. Despite the proven efficacy of CRC screening, more than one-third of African Americans have not received a colonoscopy screening within the recommended time frame (one colonoscopy per ten years). It is critical to improve this group's colonoscopy screening uptake to reduce the burden of CRC among African Americans.

Objective: The primary goal was to develop a tablet app, e-motivate, which incorporates motivational interviewing principles to increase African Americans' colonoscopy screening uptake. Two-step field testing was conducted to examine the app's efficacy.

Methods: Participants ( $\mathrm{N}=40$ ) were African American primary care patients over the age of 50 (recommended age to begin screening for CRC). Immediately after receiving a colonoscopy screening referral, patients field-tested e-motivate in the primary care office, which took approximately 20 minutes. For Field Test 1, 20 participants used the app and engaged in a think-aloud exercise to assess the intervention's feasibility. The feedback from Field Test 1 was used to modify the app. Field test procedures were repeated on an additional 20 participants to confirm feasibility. The feedback from the Field Test 2 was used to further modify the app.

Results: In Field Test 1, descriptive statistics were run to determine the usability and acceptability of the app. The mean overall score on the System Usability Scale of 86.62 (possible range from 0 to 100) indicates high usability. The mean score on the Acceptability E-Scale of 4.8 (possible range from 1 to 5) indicates high acceptability of the app. Qualitative thematic analysis revealed that participants found the e-motivate 1.0 app to be user-friendly and helpful. Some participants reported difficulty with certain app functions (e.g., using a slider icon). The participants' suggestions were used to guide the development of the e-motivate app 2.0. Field Test 2 is ongoing and results will be reported in the final poster presentation. Iterations to follow will be based on patient feedback.

Conclusions: The two-step field test approach focused on user-centered design and directly informed the development of a user-friendly, patient-driven app with optimal user satisfaction and engagement to help improve screening colonoscopy uptake in African Americans. The next critical step in the app's development is to test the efficacy of e-motivate in a randomized clinical trial. If the app is successful in the RCT there is a strong case for integrating e-motivate into standard clinical practices with the ultimate goal of reducing the preventable and unequal burden of CRC among African Americans. In the future, the digital prescribing platform RxUniverse, an efficient program which enables physicians to "prescribe" evidence-based mobile health applications to a large population of patients, can be used to bulk prescribe e-motivate.

Trial Registration: Improving Colonoscopy Screening Uptake to Reduce the Burden of CRC Among African Americans

(iproc 2017;3(1):e45) doi: 10.2196/iproc.8462

\section{KEYWORDS}

Colorectal cancer; Mobile application; Patient engagement; Prescribing 
Figure 1. Screenshot from the E-Motivate App Interface.

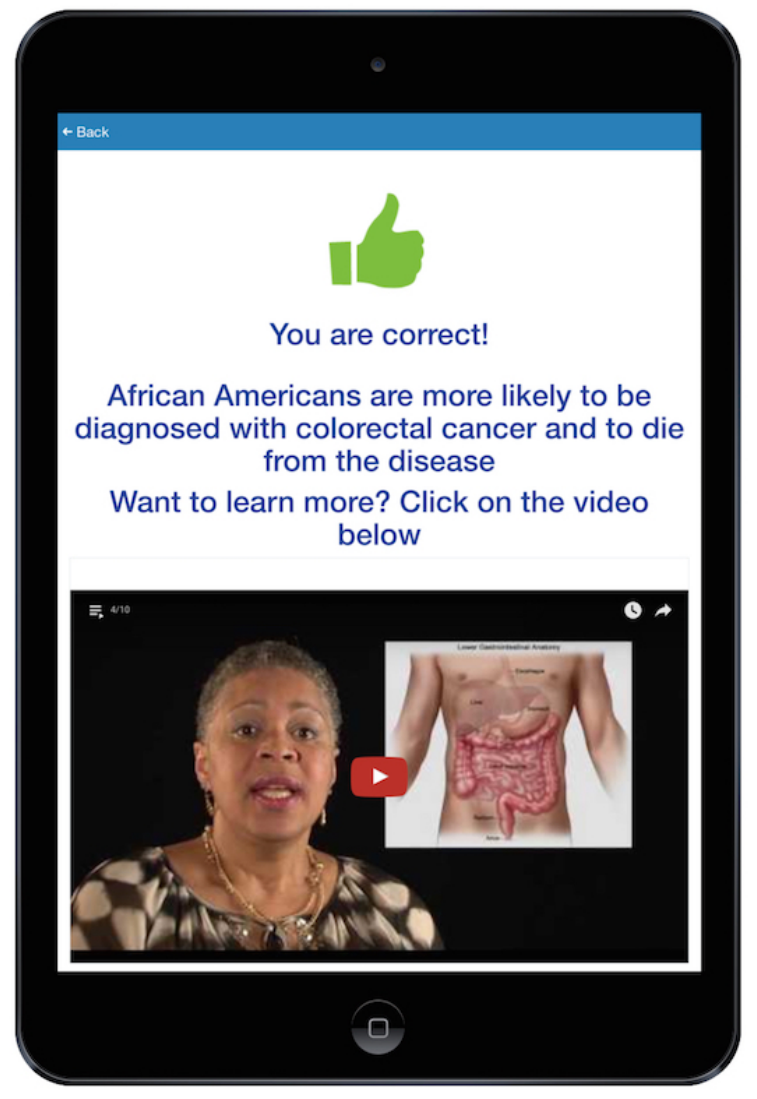

Figure 2. Screenshot from the E-Motivate App Interface.

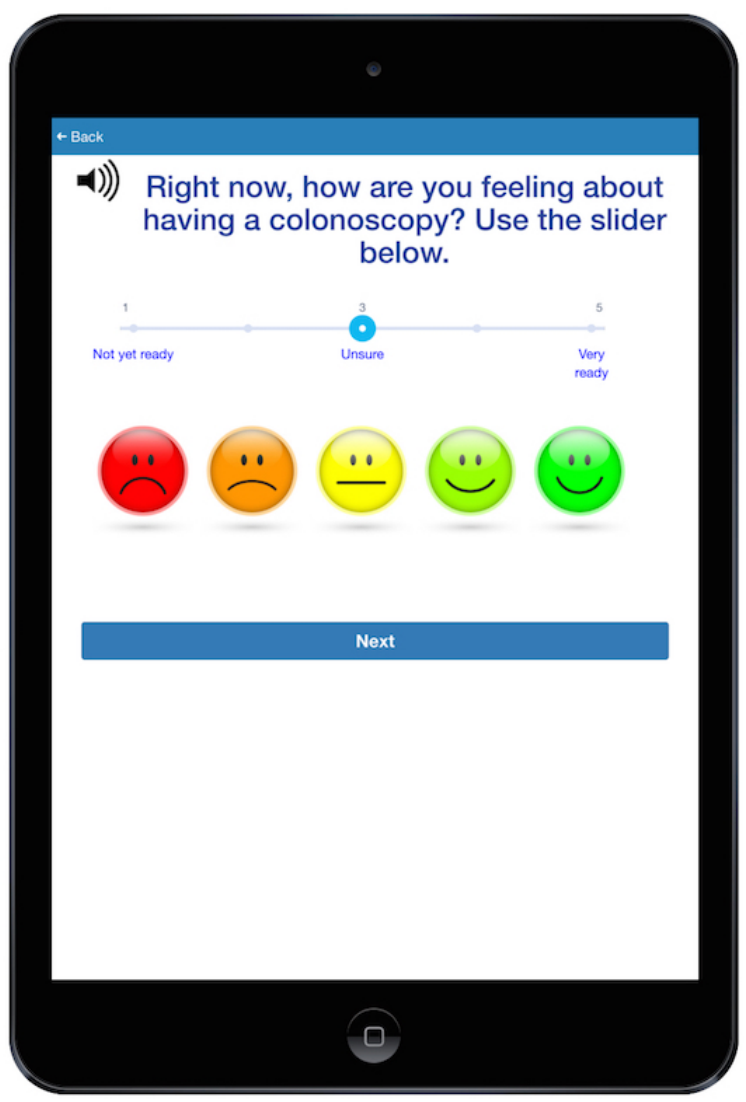




\section{Multimedia Appendix 1}

Full poster.

[PDF File (Adobe PDF File), 6MB-Multimedia Appendix 1]

Edited by T Hale; this is a non-peer-reviewed article. Submitted 13.07.17; accepted 24.08.17; published 22.09.17.

Please cite as:

Miller S, Atreja A, Fasihuddin F, Ramireddy K, Zlatopolsky R, Deorocki A, Hassanzadeh N, Farahani A, Lodhi S, Otobo E, Rogers

$J$, Jandorf $L$

E-motivate: Development of an App To Improve African Americans' Screening Colonoscopy Rates

iproc 2017;3(1):e45

URL: http://www.iproc.org/2017/1/e45/

doi: 10.2196/iproc.8462

PMID:

(CSarah Miller, Ashish Atreja, Farah Fasihuddin, Karthik Ramireddy, Rivelle Zlatopolsky, Allyssa Deorocki, Neda Hassanzadeh, Ariana Farahani, Sameed Lodhi, Emamuzo Otobo, Jason Rogers, Lina Jandorf. Originally published in Iproceedings (http://www.iproc.org), 22.09.2017. This is an open-access article distributed under the terms of the Creative Commons Attribution License (https://creativecommons.org/licenses/by/4.0/), which permits unrestricted use, distribution, and reproduction in any medium, provided the original work, first published in Iproceedings, is properly cited. The complete bibliographic information, a link to the original publication on http://www.iproc.org/, as well as this copyright and license information must be included. 